\title{
ON FUNCTIONS SATISFYING CERTAIN DIFFERENTIAL INEQUALITIES ${ }^{1}$
}

ROBERT J. BUEHLER

1. Introduction. Tagamlitzki [10] has shown that if $f(x)$ is infinitely differentiable for $x>0\left(f \in C^{\infty}(0, \infty)\right)$ and if

$$
\left|f^{(k)}(x)\right| \leqq e^{-x}, \quad x>0, \quad k=0,1,2, \cdots,
$$

then $f(x)=c e^{-x}$. In $\$ 2$ a new proof is given using Liouville's theorem. So far as I am aware, the method of proof does not apply to any essentially more general problems, depending as it does on unique properties of the analytic function $e^{z}$. However, I have obtained a few similar results by other methods, and to establish a sufficiently broad framework to contain all cases, it is convenient to pose a somewhat indefinite general problem:

Problem A. Let $C_{1}$ be a linear space of functions; let $T$ be an operator on $C_{1}$ to $C_{1}$; and let $\|\cdot\|$ be a norm defined on $C_{1}$. When does $f \in C_{1}$ and $\left\|T^{k} f\right\| \leqq 1, k=0,1,2, \cdots$, imply that $f \in C_{2}$ where $C_{2}$ is a finite-dimensional subspace of $C_{1}$ ?

A narrower framework will also be considered:

Problem B. Let $T$ be a differential operator and let $g$ be a function such that $T g=-g$ (or $+g)$. When does $\left|T^{k} f\right| \leqq|g|, k=0,1,2, \cdots$, imply $f=c g$ ?

A very simple result of type A is obtained in $\S 3$ by taking $T$ to be a self-adjoint operator on a Hilbert space. Differential operators are natural choices for $T$, and some special cases are spelled out using Fourier and Hermite expansions. A corollary of type $B$ is given.

In $\$ 4$, I consider the possibility of generalizing results for particular operators $T$ to results for conjugate operators of the form $S T S^{-1}$. For Problem B a natural choice for $S$ is an operator which acts in effect to change the variables of the differential equation $T f=-f$. By employing this device, results are obtained for example for $T=p_{1}(x) D$ $+p_{0}(x)$ and for $T=A D^{2}+B D+C$ using respectively Tagamlitzki's theorem and a Fourier expansion.

2. A property of $e^{-x}$. Theorem $1 \mathrm{~b}$ is due to Tagamlitzki [10]. For other proofs and generalizations see Tagamlitzki $[11 ; 12]$, Boas [1], and Obrechkoff [7].

Received by the editors September 26, 1960 and, in revised form, March 9, 1961.

${ }^{1}$ This work was carried out under a University of Wisconsin Fellowship. I wish to thank Professor R. C. Buck for many helpful suggestions. 
THEOREM 1a. If $f(z)$ is entire, $\left\{a_{n}\right\}$ is a sequence of positive numbers tending to infinity, and

$$
\left|f^{(k)}\left(a_{n}\right)\right| \leqq e^{-a_{n}}, \quad n, k=0,1,2, \cdots,
$$

then $f(z)=c e^{-z}$, where $c$ is constant,$|c| \leqq 1$.

Proof. Taking the absolute value of the Taylor expansion about $a_{n}$ one obtains

$$
|f(z)| \leqq \sum\left|f^{(k)}\left(a_{n}\right)\right| \cdot\left|z-a_{n}\right| k / k ! \leqq e^{-a_{n}} e^{\left|z-a_{n}\right|} .
$$

As $n \rightarrow \infty, a_{n} \rightarrow \infty$, and $-a_{n}+\left|z-a_{n}\right| \rightarrow-x$ (where $z=x+i y$ ). Hence for all $z,|f(z)| \leqq e^{-x}$. Putting $g(z)=e^{z} f(z),|g(z)|=\left|e^{z} f(z)\right|=e^{x}|f(z)|$ $\leqq 1$. By Liouville's theorem, $g(z)$ is a constant, and $f(z)=c e^{-z}$.

Theorem 1b. If $f \in C^{\infty}(0, \infty)$ and $\left|f^{(k)}(x)\right| \leqq e^{-x}$ for $x>0$, $k=0,1,2, \cdots$, then $f(x)=c e^{-x}$.

Proof. The inequalities imply that the Taylor series about an arbitrary point $a>0$ converges to $f$; hence the same proof applies by extending $f$ to an entire function.

\section{Some results for self-adjoint operators.}

TheOREM 2. Let $T$ be a self-adjoint operator on a Hilbert space $C_{1}$. Let $\left\{v_{n}\right\}$ be a complete orthonormal set such that $T v_{n}=\lambda_{n} v_{n}$. If for $M>0$, $f \in C_{1}$ satisfies

$$
\left\|T^{n} f\right\| \leqq M, \quad n=n_{k}, \quad n_{k} \rightarrow \infty,
$$

then $f=\sum_{n \in B}\left(f, v_{n}\right) v_{n}$ where $n \in B$ if and only if $\left|\lambda_{n}\right| \leqq 1$.

The proof can easily be supplied. Some special cases are given in Theorems 3, 4 and 5, using properties of Fourier and Hermite expansions.

TheOREM 3. Let $C_{1}=\left\{f: f \in C^{\infty}(-\infty, \infty)\right.$ and $f$ is periodic with period $2 \pi\}$. If $f \in C_{1}$ and if for some fixed $\mu, M$,

$$
\int_{-\pi}^{\pi}\left|D^{n} f\right|^{2} d x \leqq M \mu^{2 n}, \quad n=n_{k}, \quad n_{k} \rightarrow \infty,
$$

then $f=\sum_{-[\mu]}^{[\mu} a_{n} e^{i n x}$.

Proof. In Theorem 2 take $(f, g)=\int_{-\pi}^{\pi} f \bar{g} \cdot d x, v_{n}=(2 \pi)^{-1 / 2} e^{i n x}$, $T_{n}=i \mu^{-1} D, \lambda_{n}=n / \mu$.

CoRollary 3-1 (CARMichael [4]). Let $f(x)$ be an entire function of exponential type $\leqq q$ and let it have period $\alpha$. Let $m=[|\alpha| q / 2 \pi]$. Then $f(x)=\sum_{-m}^{m} a_{n} \exp (2 \pi i n x / \alpha)$. 
Proof. Let $f(x)=g(2 \pi x / \alpha)$. Then $g$ is of exponential type $\leqq b$ $=|\alpha| q / 2 \pi$, and has period $2 \pi$. By Theorem 2.1 of Carmichael [4] for every $\epsilon>0,\left|g^{(n)}(x)\right| \leqq M(b+\epsilon)^{n} \exp ((b+\epsilon)|x|)$ for $n=0,1,2, \cdots$, and for some $M$ independent of $n$ and $x$. Theorem 3 applies with $\mu=b+\epsilon$, and the result follows on letting $\epsilon \rightarrow 0$.

In Theorem 4, the arbitrary interval length $l$ is useful later in Corollary 7-1. Notice that the equations have a much simpler form when $l=\pi$.

Theorem 4. Let $C_{1}=\left\{f: f \in C^{\infty}[0, l]\right.$ and $f^{(2 n)}(0)=f^{(2 n)}(l)=0$, $n=0,1,2, \cdots$. If for some fixed $M>0, \mu>0$,

$$
\int_{0}^{l}\left|\left\{D^{2}+(\pi / l)^{2}-1\right\}^{n} f\right|^{2} d x \leqq M \mu^{2 n}, \quad n=n_{k}, \quad n_{k} \rightarrow \infty,
$$

then $f(x)=\sum_{1}^{[b]} a_{n} \sin (n \pi x / l)$ where $b^{2}=1+(l / \pi)^{2}(\mu-1)$.

Proof. Theorem 2 applies with

$$
\begin{aligned}
(f, g) & =\int_{0}^{l} f \bar{g} \cdot d x, & T & =\mu^{-1}\left\{D^{2}+(\pi / l)^{2}-1\right\}, \\
v_{n} & =(2 / l)^{1 / 2} \sin (n \pi x / l), & \lambda_{n} & =\mu^{-1}\left\{\left(n^{2}-1\right)(\pi / l)^{2}+1\right\} .
\end{aligned}
$$

Taking $l=\pi$ and proceeding as in Corollary $3-1$, one obtains the following result of Schoenberg [8].

COROLLARY 4-1. The only functions of exponential type satisfying $f^{(2 n)}(0)=f^{(2 n)}(\pi)=0, n=0,1,2, \cdots$, are the finite sine polynomials $f=\sum_{0}^{k} a_{n} \sin (n \pi x)$ (of type $k \pi$ ).

Corollary 4-2 is a weaker form of Theorem 4 which will be generalized in $\$ 5$, and which answers the requirements of Problem $B$ of the introduction, since $f=\sin (\pi x / l)$ satisfies $\left\{D^{2}+(\pi / l)^{2}-1\right\} f=-f$.

Corollary 4-2. If $f \in C^{\infty}[0, l]$ and $\left|\left\{D^{2}+(\pi / l)^{2}-1\right\}{ }^{n} f\right| \leqq \sin (\pi x / l)$, then $f(x)=c \cdot \sin (\pi x / l)$.

Proof. Put $\mu=1$. The assumed inequalities imply both $f \in C_{1}$ and (2).

Further special cases of Theorem 2 are obtainable from polynomial expansions. I give here only the Hermite case. Similar results for Laguerre and Jacobi polynomials can be found in [3].

Theorem 5. Let $C_{1}=\{f: f$ entire, exponential type $\}$. If $f \in C_{1}$ and if for some fixed $M>0, \mu>0$,

$$
\int_{-\infty}^{\infty} e^{-x^{2}}\left|\left(D^{2}-2 x D\right)^{n} f\right|^{2} d x \leqq M \mu^{2 n}, \quad n=n_{k}, \quad n_{k} \rightarrow \infty
$$


then $f$ is a polynomial of degree $\leqq \mu / 2$.

Proof. Theorem 2 applies on taking $T=\mu^{-1}\left(D^{2}-2 x D\right)$ and $v_{n}=H_{n}=$ the normalized Hermite polynomials, which are complete for functions of exponential type (Boas and Buck [2, p. 31]). $T f \in C_{1}$ follows from Titchmarsh $\left[13\right.$, p. 265], and $T v_{n}=-(2 n / \mu) v_{n}$ follows from Szegö [9, p. 102].

4. Conjugacy of operators. Suppose one is able to define on some appropriate space of functions an operator $S$ having $C_{1}$ as its range and having a unique inverse $S^{-1}$ defined on $C_{1}$. Then any answer to Problem A for a particular $T$ yields another for the conjugate operator $S T S^{-1}$ by Lemma 1 .

Lemma 1. Let $L=S T S^{-1}$. Let $S C_{1}=\left\{f: S^{-1} f \in C_{1}\right\}$. Define a norm $\|\cdot\|_{s}$ on $S C_{1}$ by $\|f\|_{S}=\left\|S^{-1} f\right\|$. If $f \in C_{1}$ and $\left\|T^{n} f\right\| \leqq 1$ implies $f \in C_{2}$, then $f_{1} \in S C_{1}$ and $\left\|L^{n} f_{1}\right\|_{S} \leqq 1$ implies $f_{1} \in S C_{2}$.

Proof. $1 \geqq\left\|L^{n} f_{1}\right\|_{S}=\left\|\left(S T S^{-1}\right)^{n} f_{1}\right\|_{S}=\left\|S T^{n} S^{-1} f_{1}\right\|_{S}=\left\|S^{-1} S T^{n} S^{-1} f_{1}\right\|$ $=\left\|T^{n} S^{-1} f_{1}\right\|$. Therefore $S^{-1} f_{1} \in C_{2}$, or $f_{1} \in S C_{2}$.

Possible choices for $S$ include the infinite order differential operators $X$ introduced by Delsarte and Lions $[5 ; 6]$, which have as range and domain the space of entire functions. Under these operators $X$ all $m$ th order linear differential operators $L$ having leading coefficient equal to unity are conjugate to $D^{m}$-that is, $L=X D^{m} X^{-1}$ for some $X$. Inserting the Delsarte-Lions operators $X$ for $S$ in Lemma 1, one obtains at least formal generalizations of Theorems 4 and 5 ; but whether the results can be put into any particularly meaningful or interesting form I do not know.

A type of operator $S$ which applies more naturally to Problem B arises from consideration of familiar transformations of linear differential equations. The equation $T f \equiv\left\{\sum p_{i}(x) D^{i}\right\} f=0$ is transformed into another linear differential equation by substituting $(t, v)$ for $(x, f)$ where

$$
v(x)=\phi(x) f(x), \quad t=u(x) .
$$

It can be shown that (3) is in fact the most general transformation of the form $v=g(x, f), t=h(x, f)$ which preserves linearity; thus it is a natural one to consider. Restricting $\phi$ and $u$ to $C^{\infty}$, and $u$ to be realvalued, the transformation ( 3 ) is formalized by what might be called a "multiplication operator" $\phi$ and a "substitution operator" $u$ defined by

$$
(\phi f)(x)=\phi(x) f(x), \quad(u f)(x)=f(u(x))
$$


If $\phi$ is nonzero and $u$ is monotone, then the inverses $1 / \phi$ and $u^{-1}$ exist. It is easily verified that $\phi$ and $u$ are linear operators and that they satisfy commutation rules such as

$$
D \phi=\phi D+\phi^{\prime}, \quad D u=u^{\prime} u D, \quad u \phi=\phi_{u} u
$$

where $\phi_{u}(x)=\phi(u(x))$.

We now consider the class of operators $S=\phi u$. In discussing the conjugacy relation $L=S T S^{-1}$, some attention is needed for domains of definition: if $T$ is defined on $C^{\infty}(u(a), u(b))$, then $L$ is defined on $C^{\infty}(a, b)$. Lemma 2 shows how solutions to Problem B can be generalized.

Lemma 2. Let $L=S T S^{-1}$ where $S=\phi u$, and let $h=S g$ where $T g= \pm g$. Then: (i) $L h= \pm h$, and (ii) if $f \in C_{1}$ and $\left|T^{n} f\right| \leqq|g|$ for $u(a)<x<u(b)$ implies $f \in C_{2}$, then $f \in S C_{1}$ and $\left|L^{n} f_{1}\right| \leqq|h|$ for $a<x<b$ implies $f_{1} \in S C_{2}$.

Proof. (i) $L h=S T S^{-1} h=S T S^{-1} S g=S T g= \pm S g= \pm h$. (ii) Define $\|f\|=\sup _{u(a)<x<(b)}|f / g|$. The result follows from Lemma 1 on writing $\|f\|_{S}=\left\|S^{-1} f\right\|=\sup _{u(a)<x<u(b)}\left|\left(u^{-1} \phi^{-1} f\right) / g\right|=\sup _{a<x<b}\left|\phi^{-1} f / u g\right|$ $=\sup _{a<x<b}|f / \phi u g|=\sup _{a<x<b}|f / h|$.

To what extent are linear differential operators conjugate under the operators $S=\phi \boldsymbol{u}$ ? Lemma 3 gives an answer for first order operators.

Lemma 3. Let $L=p_{1}(x) D+p_{0}(x)$, where $p_{1}, p_{0} \in C^{\infty}(a, b)$, and $0<p_{1}<\infty$ for $a<x<b$. Then $L$ is conjugate to $D$.

Proof. By commuting the operators, $S D S^{-1}=\phi u D u^{-1} \phi^{-1}$ $=\left(1 / u^{\prime}\right)\left(D-\phi^{\prime} / \phi\right)$. The requirements $p_{1}=1 / u^{\prime}$ and $p_{0}=-\phi^{\prime} / u^{\prime} \phi$ are satisfied by putting

$$
u(x)=\int_{a^{\prime}}^{x}\left(1 / p_{1}\right) d x \text { and } \phi(x)=\exp \left(-\int_{a^{\prime}}^{x}\left(p_{0} / p_{1}\right) d x\right)
$$

for an arbitrary $a^{\prime}$ in $(a, b)$. (Using $a^{\prime}$ in place of $a$ avoids difficulties when $a$ is a singularity.)

Lemma 4, concerning second order operators, will not actually be used, but it is suggestive of a classification scheme for second order differential equations.

Lemma 4. Let $T=p_{2}(x) D^{2}+p_{1}(x) D+p_{0}(x)$ where $p_{0}, p_{1}, p_{2} \in C^{\infty}(a, b)$ and $0<p_{2}<\infty$ for $a<x<b$. There is a function $q(x)$ such that $T$ is conjugate to $D^{2}+q$. Moreover $q$ is unique up to translations and reflections of the $x$ axis. 
The proof (given in [3]) is similar to that of Lemma 3. The transformation is $D^{2}+q=\phi u T u^{-1} \phi^{-1}$ where

$$
u^{-1}(x)=\int_{c}^{x} p_{2}^{-1 / 2} d x, \quad \phi(x)=\exp \int_{c}^{x}\left\{\left(p_{1, u}-u^{\prime \prime}\right) / 2 u^{\prime}\right\} d x
$$

giving $q=-\left(\phi^{\prime \prime} / \phi\right)+p_{0, u}$, where $p_{i, u}(x)=p_{i}(u(x))$.

When $T$ has constant coefficients, the canonical form is unique. The operators $4 D^{2}, 4 D^{2}+4$, and $4 D^{2}+4 D+4$, for example, are conjugate respectively to $D^{2}, D^{2}+4\left(\right.$ not $\left.D^{2}+1\right)$ and $D^{2}+3$.

5. More general answers to Problem B. Tagamlitzki's theorem may now be generalized by means of Lemmas 2 and 3 .

Theorem 6. Let $f \in C^{\infty}(a, b)$. Let $L=p_{1} D+p_{0}$ where $p_{0}, p_{1} \in C^{\infty}(a, b)$ and $0<p_{1}<\infty$ for $a<x<b$. Require further that $b$ be a singular point of $L$ (either $b=\infty$ or $\left.p_{1}(b)=0\right)$ such that for any $a^{\prime}$ in $(a, b)$,

$$
\int_{a^{\prime}}^{x}\left(1 / p_{1}\right) d x \rightarrow \infty \text { as } x \rightarrow b .
$$

Let $h$ satisfy $L h=-h$. Then $\left|L^{n} f\right| \leqq|h|, n=0,1,2, \cdots, a^{\prime}<x<b$, implies $f=c h$.

Proof. By Lemma 3, $L=S D S^{-1}$, where $S=\phi u$ with $\phi$ and $u$ given by (4). By part (i) of Lemma $2, L h=-h$ when $h=S g$ and $D g=-g$ (implying, of course, $g=k e^{-x}$ ). Theorem $1 \mathrm{~b}$ and part (ii) of Lemma 2 apply with $S C_{1}=C^{\infty}\left(a^{\prime}, b\right), C_{1}=C^{\infty}\left(u\left(a^{\prime}\right), u(b)\right)=C^{\infty}(0, \infty)$.

Theorem 7 of Tagamlitzki [11] corresponds to the special case $p_{0}=-1$, which gives $h=$ constant.

Next we give an analogous generalization of Corollary 4-2.

Theorem 7. Let $p_{1}, p_{0} \in C^{\infty}(a, b), 0<p_{1}<\infty$ for $a<x<b$. Define $u, l$, $L$, and $h$ by

$$
\begin{gathered}
u(x)=\int_{a}^{x}\left(1 / p_{1}\right) d x, \quad l=u(b), \quad L=\left(p_{1} D+p_{0}\right)^{2}+(\pi / l)^{2}-1, \\
h(x)=\sin \{\pi u(x) / l\} \exp \int_{a}^{x}\left(-p_{0} / p_{1}\right) d x
\end{gathered}
$$

Then: (i) $L h=-h$, and (ii) $\left|L^{n} f\right| \leqq|h|$ for $a<x<b$ implies $f=c h$.

Proof. Taking $S=\phi u$ from (4) one has by Lemma $3,\left(p_{1} D+p_{0}\right)^{2}$ $=\left(S D S^{-1}\right)^{2}=S D^{2} S^{-1}$. Hence $L=S T S^{-1}$ where $T=D^{2}+(\pi / l)^{2}-1$. Since $T g=-g$ when $g=\sin (\pi x / l)$, (i) follows from Lemma 2; and 
(ii) is the result of applying Lemma 2 to Corollary 4-2.

The operator $L$ of Theorem 7 includes as special cases operators having constant coefficients given by

$$
L=A D^{2}+B D+C \text { where } 1+C-B^{2} / 4 A>0,
$$

the inequality by virtue of $(\pi / l)^{2}>0$. An equivalent restriction is that $L h=-h$ has an oscillatory general solution, namely,

$$
h(x)=c_{1} e^{-B x / 2 A} \sin \left\{\pi\left(x-c_{2}\right) / A^{1 / 2} l\right\}
$$

where

$$
l^{2}=\pi^{2}\left(1+C-B^{2} / 4 A\right)^{-2} .
$$

COROLlary 7-1. Let $L$ be given by (5), and let $h$ be any solution (6) of $L h=-h$. If $\left|L^{n} f\right| \leqq|h|$ for $n=0,1,2, \cdots$, and for all $x$ between any two zeros of $h$, then $f=c h$.

Proof. In the theorem put $p_{1}=A^{1 / 2}, p_{0}=B / 2 A^{1 / 2},(a, b)=$ interval between the given zeros of $h$.

\section{BiBLIOGRAPHY}

1. R. P. Boas, Sur les suites verifiant des inegalites portant sur leurs differences, C. R. Acad. Sci. Paris 224 (1947), 1683-1685.

2. R. P. Boas and R. C. Buck, Polynomial expansions of analytic functions, Springer, Berlin, 1958.

3. R. J. Buehler, On functions satisfying certain differential inequalities, Ph.D. thesis, Univ. of Wisconsin, Madison, Wis., 1951.

4. R. D. Carmichael, Summation of functions of a complex variable, Ann. of Math. (2), 34 (1933), 349-378.

5. J. Delsarte and J. L. Lions, Transmutations d'operateurs differentiels dans le domaine complexe, C. R. Acad. Sci. Paris 244 (1957), 832-834.

6. - Transmutations d'opérateurs differentiels dans le domaine complexe, Comment. Math. Helv. 32 (1957), 113-128.

7. N. Obrechkoff, Sur les solutions bornées de quelques equations integrales singulieres, C. R. Acad. Sci. Paris 224 (1947), 993-995.

8. I. J. Schoenberg, On certain two point expansions of integral functions of exponential type, Bull. Amer. Math. Soc. 42 (1936), 284-288.

9. G. Szegö, Orthogonal polynomials, Amer. Math. Soc. Colloq. Publ. Vol. 23, Amer. Math. Soc., New York, 1939.

10. Y. Tagamlitzki, Sur les suites vérifiant certaines inégalites, C. R. Acad. Sci. Paris 223 (1946), 940-942.

11. - Funktionen, die auf der reellen Achse gewissen Ungleichungen genügen, Annuaire [Godišnik] Univ. Sofia Fac. Phys-Math. Livre 1. 42 (1946), 239-256.

12. - Sur un propriete de la fonction exponentielle, C. R. Acad. Bulgare Sci. Math. Nat. 1 (1948), 33-34.

13. E. C. Titchmarsh, The theory of functions, Oxford University Press, London, 1949.

\section{IOWA STATE University}

